\title{
The Potential Role of Probiotics in the Management of Childhood Autism Spectrum Disorders
}

\author{
J. William Critchfield, ${ }^{1}$ Saskia van Hemert, ${ }^{2}$ Michael Ash, ${ }^{3}$ \\ Linda Mulder, ${ }^{2}$ and Paul Ashwood ${ }^{1}$ \\ ${ }^{1}$ Department of Medical Microbiology and Immunology, University of California, Davis, CA 95616, USA \\ ${ }^{2}$ R\&D Department, Winclove Bio Industries, Hulstweg 11, 1032 LB Amsterdam, The Netherlands \\ ${ }^{3}$ Integrative Health Consulting, Milber, Newton Abbot, Devon TQ12 4SG, UK
}

Correspondence should be addressed to Saskia van Hemert, saskiavanhemert@winclove.nl

Received 31 March 2011; Accepted 20 August 2011

Academic Editor: P. Enck

Copyright ( 2011 J. William Critchfield et al. This is an open access article distributed under the Creative Commons Attribution License, which permits unrestricted use, distribution, and reproduction in any medium, provided the original work is properly cited.

Gastrointestinal (GI) dysfunction has been reported in a substantial number of children with autism spectrum disorders (ASD). Activation of the mucosal immune response and the presence of abnormal gut microbiota are repeatedly observed in these children. In children with ASD, the presence of GI dysfunction is often associated with increased irritability, tantrums, aggressive behaviour, and sleep disturbances. Moreover, modulating gut bacteria with short-term antibiotic treatment can lead to temporary improvement in behavioral symptoms in some individuals with ASD. Probiotics can influence microbiota composition and intestinal barrier function and alter mucosal immune responses. The administration of probiotic bacteria to address changes in the microbiota might, therefore, be a useful novel therapeutic tool with which to restore normal gut microbiota, reduce inflammation, restore epithelial barrier function, and potentially ameliorate behavioural symptoms associated with some children with ASD. In this review of the literature, support emerges for the clinical testing of probiotics in ASD, especially in the context of addressing GI symptoms.

\section{Introduction}

Autism spectrum disorders (ASD) are serious developmental disorders with soaring prevalence, affecting 1 in 91 children in the United States [1]. ASD are characterised by a spectrum of symptoms, including decreased verbal skills and social withdrawal, repetitive behavior, insistence to routines, and unusual response to sensory stimuli. Available treatments currently include specialized behavioral interventions [2] and pharmacologic interventions to assist in comorbid or associated symptoms [3]. With the underlying causes of ASD still largely undefined, the etiology of ASD is currently the focus of intensive study, with particular areas of interest including the role of environmental, genetic, and epigenetic factors $[4,5]$. These efforts share a common goal of developing new insights which can be translated into novel, effective strategies for the prevention and treatment of ASD.
The diagnosis of ASD is reached by observation of behavior and the application of well-characterized instruments, such as the Autism Diagnostic Interview-Revised (ADI-R), and the Autism Diagnostic Observation Schedule-Generic (ADOS-G). There is great heterogeneity in the individuals that receive the ASD diagnosis. This heterogeneity is not necessarily due to the diagnostic instruments but because the behaviors displayed by a group of individuals with ASD likely arise from a variety of causes. However, the causes of ASD are largely unknown, except in the rare instances in which identifiable genetic abnormalities give rise to a syndrome with ASD features. Experimental approaches seeking to obtain new insights into the etiology of ASD are increasingly focused on the selection of subgroups of ASD individuals who share a defining group of characteristics. By taking this approach, it is possible that specific factors associated with certain aspects of ASD can more readily be identified. For example, a 
prominent subgroup of individuals with ASD suffers from gastrointestinal (GI) dysfunction that stems from yet unknown causes.

The prevalence of GI symptoms in ASD is substantial, with common syptoms including diarrhea, constipation, vomiting/reflux, abdominal pain/discomfort, gaseousness, and unusually foul-smelling stools [6-8]. Individuals with ASD who have GI symptoms, compared to those without GI symptoms, often display significantly higher measures of irritability, anxiety, and social withdrawal [8]. While these studies illustrate that GI symptoms are present, the pathophysiology of these symptoms is not well understood. Despite the current lack of evidence surrounding a known GI pathology in ASD, almost one-fifth of the physicians encourage the use of probiotics for children with ASD, according to a recent survey in the United States [9]. In addition, almost 60 percent of the physicians accept the use of probiotics when the family is already utilizing them. Thus, the purpose of this paper is to put forth whether there is a case for clinical testing of probiotic preparations in selected groups of children with ASD. Rationale will be presented for the relevance of probiotic use not only in groups with GI symptoms but also in light of other immunologic and intestinal abnormalities identified in the ASD population.

Probiotics are living nonpathogenic microorganisms, which beneficially affect the host's health, when administered in adequate amounts as food ingredient or supplement. Probiotic products consist mainly of lactic acid-producing bacteria, such as lactobacilli, lactococci, and bifidobacteria, or yeasts such as Saccharomyces boulardii. The wellestablished health effects of probiotics vary with the species and particular strain(s) of bacteria chosen, based on genetic differences and the nature of bacteria-host interactions [10]. Probiotics have been used safely in the fermentations of food products for decades. Therefore, the United States Food and Drug Administration has designated probiotics as Generally Recognized as Safe (GRAS), and the European authorities provide a list of bacterial species with a Qualified Presumption of Safety (QPS).

Research on probiotics has shown efficacy of probiotic bacteria in a wide variety of health problems. Gastrointestinal difficulties such as antibiotic-associated and acute infectious diarrhea, inflammatory bowel disease (IBD), and irritable bowel syndrome (IBS) have been shown to be responsive to probiotics. In addition, probiotics have also shown activity in influencing the host's immune system [11, 12]. With the growing research on probiotics and the integral part they play in the health of not just the GI tract but the body as a whole, their inclusion as an integrative treatment may be of use for children with ASD.

\section{The GI Tract in ASD}

A notable proportion of children with ASD suffer from gastrointestinal (GI) symptoms stemming from unknown causes. The precise prevalence of GI symptoms in children with autism is not clear, with estimates ranging from between 9 to $70 \%$ [13]. The discrepancy of figures may reflect the studies reporting these estimates and range from lower estimates in some population studies to higher estimates seen in specialized GI clinics. Moreover, the assessment of GI symptoms and what constitutes GI symptoms accounts for much variation in these estimates. The fact that GI symptoms are a significant problem in autism is not in dispute but is often understated. Since GI symptoms in ASD may be important contributors to behavioral difficulties [13], carefully designed studies that investigate the full range of symptoms described for this ASD cohort are required to establish prevalence values with greater confidence. In agreement with the idea that the severities of GI symptoms and autistic symptoms could possibly be linked, Nikolov et al. reported that children with ASD and GI symptoms, compared to those without GI symptoms, display significantly higher measures of irritability, anxiety, and social withdrawal [8]. More recently, Adams et al. studied 58 children with ASD, measuring both GI symptoms and the severity of autism [14]. Their data showed a strong positive association of autism severity with GI dysfunction. This association was evident within each of the subcategories of their autism assessment, including speech, social, sensory/cognitive, and physical/ behavioural. This important new finding, while not defining a cause-effect relationship, nevertheless emphasizes the importance of developing new approaches to relieve GI symptoms in children with autism.

The GI symptoms associated with ASD are remarkably similar to those of irritable bowel syndrome (IBS) which affects $10-20 \%$ of the US population. IBS is characterized by abdominal pain/discomfort which sometimes improves upon defecation and is associated with changing stool frequency and/or changing stool consistency [15]. In a given individual, constipation or diarrhea (or neither) may predominate. Other symptoms which can accompany IBS (as well as ASD) include bloating, excess gas, gastric reflux, and food sensitivities. A careful evaluation of the patient as set forth in the ROME III criteria for functional bowel disorders [15] provides the diagnosis of IBS; this approach derives from the fact that, like ASD, there is no known biological signature or biomarker for IBS. In another parallel to ASD, IBS patients commonly have extraintestinal comorbid conditions, some of which suggest a CNS component. These include sleep difficulties, anxiety, depression, headache, and fatigue $[16,17]$. Due to the similarity of GI symptoms between ASD and IBS, an established IBS symptom scoring system has been employed in the assessment of GI symptoms in ASD [18].

Based on possible similarities between IBS and GI symptomatology in ASD, it is pertinent to note the recent successes in the treatment of IBS with probiotics. Specifically, a recent meta-analysis [19] of clinical trials highlighted two well-designed studies reporting that Bifidobacterium infantis can significantly reduce GI symptoms in IBS. In the first study, 77 subjects with IBS were evaluated over a 4 week period for GI symptoms and stool patterns, then randomized to one of three blinded treatments: Lactobacillus salivarius, Bifidobacterium infantis 35624, or placebo [20]. Symptoms including distention/bloating, difficulty with bowel movements, and abdominal discomfort/pain dropped significantly in the B. infantis group, compared to placebo, during 
the subsequent 8 week intervention period. These changes took place quickly, being evident by the end of the first week of treatment and essentially maximal by two weeks. In a larger multicenter follow-up study focusing solely on $B$. infantis 35624,362 subjects recruited in a primary care setting underwent a two-week baseline observation period then randomization to one of three encapsulated doses of $B$. infantis or placebo for a six-week intervention period [21]. Confirming and extending results of the first study, treatment with $B$. infantis $\left(10^{8} \mathrm{cfu} /\right.$ day) was associated with marked improvements such as lessening of (1) bloating/distention, (2) abdominal pain/discomfort, (3) passage of gas, (4) straining with defecation, and (5) sense of incomplete evacuation. Other improvements included scores for (1) bowel habit satisfaction and (2) global self-assessment of IBS relief. The improvements in this study were observed after approximately four weeks, in contrast to two weeks in the first study, with the slower response attributed to the encapsulated formulation which may be a less-efficient delivery system. In conclusion, clear parallels between GI symptoms in ASD and IBS, along with successes in treating IBS symptoms with probiotics, make it clear that clinical trials with probiotics in children with ASD who have GI symptoms are warranted.

\section{Microbiota Abnormalities}

Some studies suggest that children with ASD have disturbances of their intestinal microbiota compared to typically developing children. In the feces of children with ASD versus healthy controls, 10 -fold higher levels of Clostridium species were reported, with a greater diversity observed in the Clostridium species [22]. However, a comparison group consisting of ASD patients without GI symptoms was not included, so the relationship between Clostridium species and GI symptoms could not be clarified. A subsequent study revealed that there were elevations in three different Clostridium groups; Clostridium bolteae and Clostridium clusters I and XI, in individuals with ASD, but unfortunately this study did not report on the frequency of GI symptoms in the subjects [23]. More recently, Parracho et al. [24] showed that the fecal content of the Clostridium histolyticum group, known toxin producers [25], was elevated in ASD children compared to healthy unrelated controls but not compared to healthy siblings. The study included children with ASD with and without GI symptoms and found that within the ASD group high levels of Clostridium species were significantly associated with GI problems. Moreover, in a recent pyrosequencing-based study, multiple differences in fecal microbiota composition were observed among children with ASD and typically developing controls [26]. These changes included shifts at the phylum level toward a higher proportion of Bacteroidetes and a lower level of Firmicutes in autism. At the genus level, several Bifidobacterium species were lower in autism while Desulfovibrio genus was more highly represented. In general, the sibling control group was similar to the ASD group, and it was suggested that this could result from the transfer of microbial species from ASD children to unaffected siblings and may represent a risk factor for ASD rather than the result of ASD pathology. A recent culture-based assessment of fecal species in autistic children supports the finding of lower Bifidobacteria levels [14]. Finally, metabolic phenotypes, some of which can be influenced by shifts in gut microbial populations, can now be assessed via measurement of key metabolites in urine. In the context of autism, Yap et al. have defined intriguing urinary changes related to amino acid and microbial metabolites [27]. The relationship, if any, of these altered patterns to the gut microbiota in autism remains to be established.

The hypothesis that a gut microbial imbalance, such as the presence of toxin-producing Clostridium species, could contribute to ASD behavioral symptoms was addressed in a small study consisting of 11 children with chronic diarrhea and a late-onset phenotype of ASD [28]. Subjects were treated for 8 weeks with vancomycin, a strategic drug in the treatment of chronic Clostridium difficile diarrhea. Scores for behavior and communication improved significantly during the treatment period; however, these gains in behavior were temporarliy and deteriorated in most cases after termination of the treatment. Unfortunately, GI symptomatology and fecal flora composition were not reported, and the experimental design suffered from a lack of a control group. In spite of these shortcomings, this study is the only one of its kind and suggests that ASD symptoms in a selected group of children may have a relationship to the composition of intestinal bacterial species that are sensitive to vancomycin.

Disruption of the balance that exists between different enteric microbiota populations may predispose an individual to altered gut motility and secretion, which results in diarrhea or constipation, symptoms commonly reported in autistic patients. These changes are, in turn, likely to influence the balance of enteric microbiota. Probiotics can be useful for maintaining or restoring the balance of the intestinal microbiota [29]. For example, a probiotic mixture has been shown to affect both the composition as well as the metabolic activity of the fecal microbiota in healthy volunteers taking the antibiotic amoxicillin [30]. In addition, specific strains of probiotics have been shown to inhibit the growth of different Clostridium species [31-34]. There is also good in vivo evidence that probiotics can be useful for the treatment of Clostridium difficile infections (for a review, see [35]). By analogy, the testing of probiotics in ASD could reveal whether they are able to normalize gut microbial populations, reduce GI symptoms (in those affected), and alleviate behavioral difficulties.

\section{Mucosal Immunity}

Several studies have sought to determine whether there is evidence of intestinal pathology in ASD. Findings of significant but subtle widespread gastrointestinal pathology have been noted in children with ASD who have GI symptoms sufficient to warrant clinical followup [6, 13, 36]. However, the existence of a gastrointestinal pathology specific to persons with ASD is a controversial topic. Histology, immunohistochemistry, and flow cytometry evidence has consistently shown a subtle, panenteric infiltration of immune cells such as lymphocytes, monocytes, NK cells, and eosinophils into the walls of the GI tract in some children 
with ASD, compared with typically developing children with GI symptoms [37-42]. Furthermore, the infiltrating lymphocytes exhibit a marked proinflammatory phenotype with increased $\mathrm{CD}^{+}{ }^{+} \mathrm{TNF}^{+}$cells, $\mathrm{CD}^{+}$IFN $\gamma^{+}$cells and reduced regulatory $\mathrm{CD}^{+}{ }^{+} \mathrm{IL}-10^{+}$cells in ASD children with GI symptoms [38, 39]. The observatons, thus far made, do not suggest that any pathology is similar to known inflammatory conditions such as Crohn's disease, ulcerative colitis, or celiac disease. Furthermore, intestinal immunological abnormalities can also be found in children with typical development, as well as children with food allergies and immunodeficiencies [13]. By using two inflammatory markers in the feces, which are increased in some IBD patients, there was no evidence found for a link between active (i.e., neutrophil related) inflammation in the gut and autism [43]. Thus, despite evident symptomatology there remains significant contention as to whether this relates to GI pathophysiology in ASD, with many proponents and detractors [44].

Healthy individuals have an intact intestinal barrier, essential in maintaining health and preventing tissue injury [45]. Despite the arguments surrounding gut pathology in ASD, several studies have shown that the integrity of the intestinal mucosal barrier might be compromised in ASD [36]. The intestinal barrier quality can be measured by urinary levels of lactulose and mannitol postoral challenge. This allows calculation of a lactulose:mannitol ratio, which is a good marker of small intestinal permeability [45]. Intestinal permeability, measured by the lactulose:mannitol test, has been shown to be increased in autistic children compared to healthy controls [46-48]. Furthermore, colocalization of immunoglobulin G/complement C1q deposition on the basement membrane of epithelium of the GI tract is observed in ASD children, suggesting the presence of inflammatory processes and/or an autoimmune component that may affect the integrity of the mucosal barrier [41, 42]. It has been suggested that this increased permeability could lead to entering of byproducts of commensal or pathogenic bacteria as well as food-derived peptides into the blood. This entry of antigenic material may lead to immune responses that could affect neuronal signaling, or the material could directly interact with the peripheral nervous system [47, 48]. However, further testing is required [13], as the studies thus far have had methodological limitations including small subject populations. Properly powered prospective studies with appropriate controls are warranted to address the issues surrounding epithelial damage and permeability.

Probiotics are capable of stabilizing the mucosal barrier by increasing mucin expression, reducing bacterial overgrowth, stimulating mucosal immunity (secretory IgA), and synthesizing antioxidant substances [49]. Although most of these findings are based on cell culture systems and animal models, in vivo human work has also revealed enhanced expression of duodenal epithelial tight junction proteins in the context of short-term exposure to L. plantarum WCFS1 [50]. This supports the concept that probiotics can have a role in maintaining or improving gut barrier function in humans. In addition, specific probiotics have been proven to be successful in preventing the recurrence of inflammation in some situations and diseases such as IBD (for reviews see [51-53]). The controversies surrounding the extent and the nature of GI pathology in ASD exists, and studies that address the potential use of probiotics may well have to be confined to treatment of GI symptoms in ASD as opposed to clinically defined pathological criteria.

\section{Immune Dysfunction}

A possible role for immune dysfunction in ASD has been described for many years, and numerous immune abnormalities have been reported in ASD. However, results are rather variable and sometimes conflicting, due to the small number of study subjects, heterogeneous patient populations, and lack of proper controls [54]. Nevertheless, many findings point to aberrant immune activation, likely in a subset of individuals with ASD. These findings include an abnormal ratio of $\mathrm{CD}^{+}$to $\mathrm{CD} 8^{+} \mathrm{T}$-cells, abnormal or skewed T-helper-cell $\left(\mathrm{T}_{\mathrm{H}} 1 / \mathrm{T}_{\mathrm{H}} 2 / \mathrm{T}_{\mathrm{H}} 17\right)$ cytokine profiles, elevated blood monocyte counts, decreased lymphocyte numbers, self-reactive antibodies to brain and CNS proteins, neuroinflammation, imbalance of serum and mucosal immunoglobulin levels and increased nitric oxide mechanism (for reviews, see $[39,55,56])$. Immuno based therapies have largely been ignored in autism, but it has been shown that treatment with anti-inflammatory drugs induce clinical improvement in ASD [57].

A large part of the immune system (approximately $80 \%)$ is concentrated in and around the intestinal mucosa. The intestinal microbiota is involved in maturation of the immune system as demonstrated in studies in germ-free mice [58]. In turn, the microbiota in the intestines plays an important role in the regulation of functions in the immune system [59]. The immune system can be modulated by probiotic bacteria, and these effects are highly species and strain specific $[11,59,60]$ with both in vitro cytokine responses and in vivo systemic effects altered by probiotics [61-67]. The accumulation of evidence suggests that probiotics play a role in providing tolerogenic signals. For example, a role for gut microbiota in autoimmune diseases has been suggested [68] and is supported by animal models demonstrating positive effects of probiotics on autoimmune diseases $[66,69,70]$. Although not much evidence has yet been reported on the effect of probiotics in autoimmunity in humans, a pilot study indicated positive effects in rheumatoid arthritis [71]. Probiotic bacteria themselves as well as metabolites produced by a probiotic bacterium have shown to suppress the activation of MCP-1, a chemokine shown to be dysregulated in ASD [72-75]. However, it must be noted that the effects of probiotics on cytokine and chemokine responses are highly strain specific, and probiotic bacteria differ in their ability to stimulate anti-inflammatory IL-10 secretion by peripheral blood mononuclear cells (PBMC) in vitro [7678]. Bacterial strains with a good in vitro ratio of antiinflammatory cytokines versus proinflammatory cytokines have been shown to have anti-inflammatory properties in vivo [76]. In vivo administration of Lactobacillus rhamnosus GG induced higher IL-10 serum levels in allergic children [79], but this finding was not confirmed in other studies 
$[64,80]$. Thus, while probiotics have good in vitro capacities to induce anti-inflammatory cytokines, their effect on systemic cytokine profiles remains to be proven and may be dependent on the microbiota mix in situ.

It is well established that there is bidirectional communication between the gut, the immune system, and the brain. For example, psychological stress can induce changes of the gastrointestinal microbes. On the other hand, intestinal bacteria can directly communicate with the central nervous system by the way of the vagal sensory nerve fibers and the peripheral immune system [81]. Intraventricular administration of propionic acid, a metabolite produced by gut bacteria, has been shown in a rat model to change both brain and behavior in a manner that is consistent with symptoms associated with ASD; these are not "autistic features" but may have some validity with ASD $[82,83]$. It is possible that gut-brain interactions may contribute to abnormal neural development and the subsequent expression of aberrant behavior. Increased gut permeability may play an important role in the gut-brain relationship, as partially digested food and bacterial components can pass into the blood stream and may interfere directly and indirectly with the central nervous system. One of these immunoreactive compounds could be dietary-derived opioid peptides, derived from gluten and casein. Increased intestinal permeability can also permit entry of lipopolysaccharides (LPS), a potent proinflammatory compound of the cell walls of gram-negative bacteria. Leakage of lipopolysaccharides from the intestine might be the trigger for peripheral inflammatory responses that lead to de novo production of cytokines in the brain. By improving the epithelial barrier, this may reduce traffic of bacteria and their byproducts and might be a way to stop the inflammatory response. One study by Emmanuelle and colleagues showed increased LPS in the blood of individuals with ASD, a finding that corresponded to increased circulating IL-6 levels in the periphery and may reflect both an increased intestinal permeability and activation of immune responses that result in the production of this proficient neuromodulating cytokine [84].

Administration of probiotics can have influences on neuronal function, as shown by different studies. For example, administration of Bifidobacterium infantis in a rat model of depression showed effects on immune, neuroendocrine, and central monoaminergic activity [85]. The proinflammatory immune response was attenuated, and tryptophan was elevated by the bifidobacteria treatment. A probiotic drink containing Lactobacillus casei has also revealed positive effects on mood and cognition in volunteers [86]. Moreover, a pilot study in chronic fatigue patients has been performed with the same probiotic product [87]. Interestingly, almost all chronic fatigue syndrome patients report neuropsychological disturbances. The study showed a significant decrease in anxiety symptoms and suggested follow-up studies to examine anxiety and depression, including inflammatory cytokines and other immune mediators, blood tryptophan levels, and urinary metabolites of neurotransmitters [87]. Mechanisms of action by which probiotics can influence brain processes are not yet fully elucidated, but likely involve multiple pathways of the interplay between brain, gut, and immune system. Maintaining a balance between host defense and uncontrolled or unresolving inflammation relies on a mutualistic crosstalk between the immune system and the microbiome, especially in the maintenance of intestinal homeostasis, an area of relevance for a subset of ASDs [88]. Probiotics will become increasingly defined by strain-specific outcomes as this evolving area of crosscommunication between the respective genes of the host and the microbiota becomes more clearly defined. Probiotics may offer a potential therapeutic that could beneficially alter the gut-brain axis and modify aberrant behaviors related to altered immune inflammatory outputs.

\section{Conclusions and Further Perspectives}

Autism spectrum disorders are a diverse group of disorders caused by a complex interplay between genetic and environmental components. There is a range of indications that alterations in the intestinal microbiota in the gut might contribute to the disorder in a substantial number of individuals. Probiotics can be useful to restore the microbial balance in the intestine, to relieve gastrointestinal problems and to attenuate immunological abnormalities. Whether the use of probiotics by children with autism can lead to improvements in behaviors needs to be established in well-controlled trials with sufficient group sizes. Important for these trials is the choice of the bacterial strains, as effects of probiotic bacteria can be highly strain specific.

\section{References}

[1] M. D. Kogan, S. J. Blumberg, L. A. Schieve et al., "Prevalence of parent-reported diagnosis of autism spectrum disorder among children in the US, 2007," Pediatrics, vol. 124, no. 5, pp. 1395$1403,2009$.

[2] J. K. Seida, M. B. Ospina, M. Karkhaneh, L. Hartling, V. Smith, and B. Clark, "Systematic reviews of psychosocial interventions for autism: an umbrella review," Developmental Medicine and Child Neurology, vol. 51, no. 2, pp. 95-104, 2009.

[3] T. J. Leskovec, B. M. Rowles, and R. L. Findling, "Pharmacological treatment options for autism spectrum disorders in children and adolescents," Harvard Review of Psychiatry, vol. 16, no. 2, pp. 97-112, 2008.

[4] I. Hertz-Picciotto, L. A. Croen, R. Hansen, C. R. Jones, J. van de Water, and I. N. Pessah, "The CHARGE study: an epidemiologic investigation of genetic and environmental factors contributing to autism," Environmental Health Perspectives, vol. 114, no. 7, pp. 1119-1125, 2006.

[5] M. Losh, P. F. Sullivan, D. Trembath, and J. Piven, "Current developments in the genetics of autism: from phenome to genome," Journal of Neuropathology and Experimental Neurology, vol. 67, no. 9, pp. 829-837, 2008.

[6] K. Horvath and J. A. Perman, "Autism and gastrointestinal symptoms," Current Gastroenterology Reports, vol. 4, no. 3, pp. 251-258, 2002.

[7] C. A. Molloy and P. Manning-Courtney, "Prevalence of chronic gastrointestinal symptoms in children with autism and autistic spectrum disorders," Autism, vol. 7, no. 2, pp. 165-171, 2003. 
[8] R. N. Nikolov, K. E. Bearss, J. Lettinga et al., "Gastrointestinal symptoms in a sample of children with pervasive developmental disorders," Journal of Autism and Developmental Disorders, vol. 39, no. 3, pp. 405-413, 2009.

[9] A. E. Golnik and M. Ireland, "Complementary alternative medicine for children with autism: a physician survey," Journal of Autism and Developmental Disorders, vol. 39, no. 7, pp. 9961005, 2009.

[10] V. Azas-Braesco, J. L. Bresson, F. Guarner, and G. Corthier, "Not all lactic acid bacteria are probiotics, but some are," British Journal of Nutrition, vol. 103, no. 7, pp. 1079-1081, 2010.

[11] H. Gill and J. Prasad, "Probiotics, immunomodulation, and health benefits," Advances in Experimental Medicine and Biology, vol. 606, pp. 423-454, 2008.

[12] A. C. Ouwehand, S. Salminen, and E. Isolauri, "Probiotics: an overview of beneficial effects," Antonie van Leeuwenhoek, International Journal of General and Molecular Microbiology, vol. 82, no. 1-4, pp. 279-289, 2002.

[13] T. Buie, D. B. Campbell, G. J. Fuchs et al., "Evaluation, diagnosis, and treatment of gastrointestinal disorders in individuals with ASDs: a consensus report," Pediatrics, vol. 125, supplement 1, pp. S1-S18, 2010.

[14] J. B. Adams, L. J. Johansen, L. D. Powell, D. Quig, and R. A. Rubin, "Gastrointestinal flora and gastrointestinal status in children with autism - comparisons to typical children and correlation with autism severity," BMC Gastroenterology, vol. 11 , article 22, 2011.

[15] G. F. Longstreth, W. G. Thompson, W. D. Chey, L. A. Houghton, F. Mearin, and R. C. Spiller, "Functional bowel disorders," Gastroenterology, vol. 130, no. 5, pp. 1480-1491, 2006.

[16] K. Tillisch and L. Chang, "Diagnosis and treatment of irritable bowel syndrome: state of the art," Current Gastroenterology Reports, vol. 7, no. 4, pp. 249-256, 2005.

[17] E. J. Videlock and L. Chang, "Irritable bowel syndrome: current approach to symptoms, evaluation, and treatment," Gastroenterology Clinics of North America, vol. 36, no. 3, pp. 665-685, 2007.

[18] B. L. Handen, R. D. Melmed, R. L. Hansen et al., "A doubleblind, placebo-controlled trial of oral human immunoglobulin for gastrointestinal dysfunction in children with autistic disorder," Journal of Autism and Developmental Disorders, vol. 39, no. 5, pp. 796-805, 2009.

[19] D. M. Brenner, M. J. Moeller, W. D. Chey, and P. S. Schoenfeld, "The utility of probiotics in the treatment of irritable bowel syndrome: a systematic review," American Journal of Gastroenterology, vol. 104, no. 4, pp. 1033-1049, 2009.

[20] L. O’Mahony, J. Mccarthy, P. Kelly et al., "Lactobacillus and Bifidobacterium in irritable bowel syndrome: symptom responses and relationship to cytokine profiles," Gastroenterology, vol. 128, no. 3, pp. 541-551, 2005.

[21] P. J. Whorwell, L. Altringer, J. Morel et al., "Efficacy of an encapsulated probiotic Bifidobacterium infantis 35624 in women with irritable bowel syndrome," American Journal of Gastroenterology, vol. 101, no. 7, pp. 1581-1590, 2006.

[22] S. M. Finegold, D. Molitoris, Y. Song et al., "Gastrointestinal microflora studies in late-onset autism," Clinical Infectious Diseases, vol. 35, supplement 1, pp. S6-S16, 2002.

[23] Y. Song, C. Liu, and S. M. Finegold, "Real-time PCR quantitation of clostridia in feces of autistic children," Applied and Environmental Microbiology, vol. 70, no. 11, pp. 6459-6465, 2004.
[24] H. M. R. T. Parracho, M. O. Bingham, G. R. Gibson, and A. L. McCartney, "Differences between the gut microflora of children with autistic spectrum disorders and that of healthy children," Journal of Medical Microbiology, vol. 54, no. 10, pp. 987-991, 2005.

[25] C. L. Hatheway, “Toxigenic clostridia,” Clinical Microbiology Reviews, vol. 3, no. 1, pp. 66-98, 1990.

[26] S. M. Finegold, S. E. Dowd, V. Gontcharova et al., "Pyrosequencing study of fecal microflora of autistic and control children," Anaerobe, vol. 16, no. 4, pp. 444-453, 2010.

[27] I. K. S. Yap, M. Angley, K. A. Veselkov, E. Holmes, J. C. Lindon, and J. K. Nicholson, "Urinary metabolic phenotyping differentiates children with autism from their unaffected siblings and age-matched controls," Journal of Proteome Research, vol. 9, no. 6, pp. 2996-3004, 2010.

[28] R. H. Sandler, S. M. Finegold, E. R. Bolte et al., "Short-term benefit from oral vancomycin treatment of regressive-onset autism," Journal of Child Neurology, vol. 15, no. 7, pp. 429$435,2000$.

[29] L. J. Fooks and G. R. Gibson, "Probiotics as modulators of the gut flora," British Journal of Nutrition, vol. 88, no. 1, pp. S39S49, 2002.

[30] C. J. M. Koning, D. M. A. E. Jonkers, E. E. Stobberingh, L. Mulder, F. M. Rombouts, and R. W. Stockbrügger, "The effect of a multispecies probiotic on the intestinal microbiota and bowel movements in healthy volunteers taking the antibiotic amoxycillin," American Journal of Gastroenterology, vol. 103, 1, pp. 178-189, 2008.

[31] L. Bevilacqua, M. Ovidi, E. Di Mattia, L. D. Trovatelli, and F. Canganella, "Screening of Bifidobacterium strains isolated from human faeces for antagonistic activities against potentially bacterial pathogens," Microbiological Research, vol. 158, no. 2, pp. 179-185, 2003.

[32] B. Bogovič Matijašić, M. Koman Rajšp, B. Perko, and I. Rogelj, "Inhibition of Clostridium tyrobutyricum in cheese by Lactobacillus gasseri," International Dairy Journal, vol. 17, no. 2, pp. 157-166, 2007.

[33] P. Naaber, I. Smidt, J. Štšepetova, T. Brilene, H. Annuk, and M. Mikelsaar, "Inhibition of Clostridium difficile strains by intestinal Lactobacillus species," Journal of Medical Microbiology, vol. 53, no. 6, pp. 551-554, 2004.

[34] A. Y. L. Teo and H. M. Tan, "Inhibition of Clostridium perfringens by a novel strain of Bacillus subtilis isolated from the gastrointestinal tracts of healthy chickens," Applied and Environmental Microbiology, vol. 71, no. 8, pp. 4185-4190, 2005.

[35] L. V. McFarland, "Evidence-based review of probiotics for antibiotic-associated diarrhea and Clostridium difficile infections," Anaerobe, vol. 15, no. 6, pp. 274-280, 2009.

[36] J. F. White, "Intestinal pathophysiology in autism," Experimental Biology and Medicine, vol. 228, no. 6, pp. 639-649, 2003.

[37] P. Ashwood, A. Anthony, A. A. Pellicer, F. Torrente, J. A. Walker-Smith, and A. J. Wakefield, "Intestinal lymphocyte populations in children with regressive autism: evidence for extensive mucosal immunopathology," Journal of Clinical Immunology, vol. 23, no. 6, pp. 504-517, 2003.

[38] P. Ashwood, A. Anthony, F. Torrente, and A. J. Wakefield, "Spontaneous mucosal lymphocyte cytokine profiles in children with autism and gastrointestinal symptoms: mucosal immune activation and reduced counter regulatory interleukin-10," Journal of Clinical Immunology, vol. 24, no. 6, pp. 664-673, 2004.

[39] P. Ashwood and A. J. Wakefield, "Immune activation of peripheral blood and mucosal CD3+ lymphocyte cytokine 
profiles in children with autism and gastrointestinal symptoms," Journal of Neuroimmunology, vol. 173, no. 1-2, pp. 126134, 2006.

[40] R. I. Furlano, A. Anthony, R. Day et al., "Colonic CD8 and $\gamma \delta$ T-cel infiltration with epithelial damage in children with autism," Journal of Pediatrics, vol. 138, no. 3, pp. 366-372, 2001.

[41] F. Torrente, A. Anthony, R. B. Heuschkel, M. A. Thomson, P. Ashwood, and S. H. Murch, "Focal-enhanced gastritis in regressive autism with features distinct from Crohn's and helicobacter pylori gastritis," American Journal of Gastroenterology, vol. 99, no. 4, pp. 598-605, 2004.

[42] F. Torrente, P. Ashwood, R. Day et al., "Small intestinal enteropathy with epithelial IgG and complement deposition in children with regressive autism," Molecular Psychiatry, vol. 7, no. 4, pp. 375-382, 2002.

[43] E. Fernell, U. L. Fagerberg, and P. M. Hellström, "No evidence for a clear link between active intestinal inflammation and autism based on analyses of faecal calprotectin and rectal nitric oxide," Acta Paediatrica, International Journal of Paediatrics, vol. 96, no. 7, pp. 1076-1079, 2007.

[44] P. Galiatsatos, A. Gologan, and E. Lamoureux, "Autistic enterocolitis: fact or fiction?" Canadian Journal of Gastroenterology, vol. 23, no. 2, pp. 95-98, 2009.

[45] A. Farhadi, A. Banan, J. Fields, and A. Keshavarzian, "Intestinal barrier: an interface between health and disease," Journal of Gastroenterology and Hepatology, vol. 18, no. 5, pp. 479-497, 2003.

[46] L. De Magistris, V. Familiari, A. Pascotto et al., "Alterations of the intestinal barrier in patients with autism spectrum disorders and in their first-degree relatives," Journal of Pediatric Gastroenterology and Nutrition, vol. 51, no. 4, pp. 418-424, 2010.

[47] P. D’Eufemia, M. Celli, R. Finocchiaro et al., "Abnormal intestinal permeability in children with autism," Acta Paediatrica, International Journal of Paediatrics, vol. 85, no. 9, pp. 1076-1079, 1996.

[48] K. Horvath and J. A. Perman, "Autistic disorder and gastrointestinal disease," Current Opinion in Pediatrics, vol. 14, no. 5, pp. 583-587, 2002.

[49] F. Lutgendorff, L. M. A. Akkermans, and J. D. Söderholm, “The role of microbiota and probiotics in stress-induced gastrointestinal damage," Current Molecular Medicine, vol. 8, no. 4, pp. 282-298, 2008.

[50] J. Karczewski, F. J. Troost, I. Konings et al., "Regulation of human epithelial tight junction proteins by Lactobacillus plantarum in vivo and protective effects on the epithelial barrier," American Journal of Physiology, vol. 298, no. 6, pp. G851-G859, 2010.

[51] C. Hedin, K. Whelan, and J. O. Lindsay, "Evidence for the use of probiotics and prebiotics in inflammatory bowel disease: a review of clinical trials," Proceedings of the Nutrition Society, vol. 66, no. 3, pp. 307-315, 2007.

[52] K. P. Rioux and R. N. Fedorak, "Probiotics in the treatment of inflammatory bowel disease," Journal of Clinical Gastroenterology, vol. 40, no. 3, pp. 260-263, 2006.

[53] B. Sheil, F. Shanahan, and L. O'Mahony, "Probiotic effects on inflammatory bowel disease," Journal of Nutrition, vol. 137, no. 3, supplement 2, pp. 819S-824S, 2007.

[54] K. A. Stigler, T. L. Sweeten, D. J. Posey, and C. J. McDougle, "Autism and immune factors: a comprehensive review," Research in Autism Spectrum Disorders, vol. 3, no. 4, pp. 840-860, 2009.
[55] M. Careaga, J. Van de Water, and P. Ashwood, "Immune dysfunction in autism: a pathway to treatment," Neurotherapeutics, vol. 7, no. 3, pp. 283-292, 2010.

[56] A. Enstrom, P. Krakowiak, C. Onore et al., "Increased IgG4 levels in children with autism disorder," Brain, Behavior, and Immunity, vol. 23, no. 3, pp. 389-395, 2009.

[57] M. Boris, C. C. Kaiser, A. Goldblatt et al., "Effect of pioglitazone treatment on behavioral symptoms in autistic children," Journal of Neuroinflammation, vol. 4, article 3, 2007.

[58] L. V. Hooper and J. I. Gordon, "Commensal host-bacterial relationships in the gut," Science, vol. 292, no. 5519, pp. 1115$1118,2001$.

[59] V. Delcenserie, D. Martel, M. Lamoureux, J. Amiot, Y. Boutin, and D. Roy, "Immunomodulatory effects of probiotics in the intestinal tract," Current Issues in Molecular Biology, vol. 10, no. 1, pp. 37-54, 2008.

[60] E. Nova, J. Wärnberg, S. Gómez-Martínez, L. E. Díaz, J. Romeo, and A. Marcos, "Immunomodulatory effects of probiotics in different stages of life," British Journal of Nutrition, vol. 98, no. 1, pp. S90-S95, 2007.

[61] D. Bunout, S. Hirsch, M. P. De la Maza et al., "Effects of prebiotics on the immune response to vaccination in the elderly," Journal of Parenteral and Enteral Nutrition, vol. 26, no. 6, pp. 372-376, 2002.

[62] M. De Vrese, P. Rautenberg, C. Laue, M. Koopmans, T. Herremans, and J. Schrezenmeir, "Probiotic bacteria stimulate virus-specific neutralizing antibodies following a booster polio vaccination," European Journal of Nutrition, vol. 44, no. 7, pp. 406-413, 2005.

[63] M. Hisbergues, M. Magi, P. Rigaux et al., "In vivo and in vitro immunomodulation of Der $\mathrm{p} 1$ allergen-specific response by Lactobacillus plantarum bacteria," Clinical and Experimental Allergy, vol. 37, no. 9, pp. 1286-1295, 2007.

[64] L. Niers, R. Martín, G. Rijkers et al., "The effects of selected probiotic strains on the development of eczema (the PandA study)," Allergy, vol. 64, no. 9, pp. 1349-1358, 2009.

[65] D. Paineau, D. Carcano, G. Leyer et al., "Effects of seven potential probiotic strains on specific immune responses in healthy adults: a double-blind, randomized, controlled trial," FEMS Immunology and Medical Microbiology, vol. 53, no. 1, pp. 107113, 2008.

[66] J. S. So, H. K. Kwon, C. G. Lee et al., "Lactobacillus casei suppresses experimental arthritis by down-regulating $\mathrm{T}$ helper 1 effector functions," Molecular Immunology, vol. 45, no. 9, pp. 2690-2699, 2008.

[67] J. Z. Xiao, S. Kondo, N. Yanagisawa et al., "Probiotics in the treatment of Japanese cedar pollinosis: a double-blind placebo-controlled trial," Clinical and Experimental Allergy, vol. 36, no. 11, pp. 1425-1435, 2006.

[68] E. B. Canche-Pool, R. Cortez-Gómez, R. Flores-Mejía et al., "Probiotics and autoimmunity: an evolutionary perspective," Medical Hypotheses, vol. 70, no. 3, pp. 657-660, 2008.

[69] J. Ezendam, A. De Klerk, E. R. Gremmer, and H. Van Loveren, "Effects of Bifidobacterium animalis administered during lactation on allergic and autoimmune responses in rodents," Clinical and Experimental Immunology, vol. 154, no. 3, pp. 424-431, 2008.

[70] S. Lavasani, B. Dzhambazov, M. Nouri et al., "A novel probiotic mixture exerts a therapeutic effect on experimental autoimmune encephalomyelitis mediated by IL-10 producing regulatory T cells," PLoS One, vol. 5, no. 2, Article ID e9009, 2010.

[71] K. Hatakka, J. Martio, M. Korpela et al., "Effects of probiotic therapy on the activity and activation of mild rheumatoid 
arthritis-a pilot study," Scandinavian Journal of Rheumatology, vol. 32, no. 4, pp. 211-215, 2003.

[72] P. Ashwood, P. Krakowiak, I. Hertz-Picciotto, R. Hansen, I. Pessah, and J. Van de Water, "Elevated plasma cytokines in autism spectrum disorders provide evidence of immune dysfunction and are associated with impaired behavioral outcome," Brain, Behavior, and Immunity, vol. 25, no. 1, pp. 4045, 2011.

[73] Y. P. Lin, C. H. Thibodeaux, J. A. Peña, G. D. Ferry, and J. Versalovic, "Probiotic Lactobacillus reuteri suppress proinflammatory cytokines via c-Jun," Inflammatory Bowel Diseases, vol. 14, no. 8, pp. 1068-1083, 2008.

[74] E. O. Petrof, E. C. Claud, J. Sun et al., "Bacteria-free solution derived from Lactobacillus plantarum inhibits multiple NFkappaB pathways and inhibits proteasome function," Inflammatory Bowel Diseases, vol. 15, no. 10, pp. 1537-1547, 2009.

[75] D. L. Vargas, C. Nascimbene, C. Krishnan, A. W. Zimmerman, and C. A. Pardo, "Neuroglial activation and neuroinflammation in the brain of patients with autism," Annals of Neurology, vol. 57, no. 1, pp. 67-81, 2005.

[76] B. Foligne, S. Nutten, C. Grangette et al., "Correlation between in vitro and in vivo immunomodulatory properties of lactic acid bacteria," World Journal of Gastroenterology, vol. 13, no. 2, pp. 236-243, 2007.

[77] M. Medina, E. Izquierdo, S. Ennahar, and Y. Sanz, "Differential immunomodulatory properties of Bifidobacterium logum strains: relevance to probiotic selection and clinical applications," Clinical and Experimental Immunology, vol. 150, no. 3, pp. 531-538, 2007.

[78] L. E. M. Niers, H. M. Timmerman, G. T. Rijkers et al., "Identification of strong interleukin-10 inducing lactic acid bacteria which down-regulate T helper type 2 cytokines," Clinical and Experimental Allergy, vol. 35, no. 11, pp. 1481-1489, 2005.

[79] T. Pessi, Y. Sütas, M. Hurme, and E. Isolauri, "Interleukin10 generation in atopic children following oral lactobacillus rhamnosus GG," Clinical and Experimental Allergy, vol. 30, no. 12, pp. 1804-1808, 2000.

[80] M. V. Kopp, M. Goldstein, A. Dietschek, J. Sofke, A. Heinzmann, and R. Urbanek, "Lactobacillus GG has in vitro effects on enhanced interleukin-10 and interferon- $\gamma$ release of mononuclear cells but no in vivo effects in supplemented mothers and their neonates," Clinical and Experimental Allergy, vol. 38, no. 4, pp. 602-610, 2008.

[81] L. E. Goehler, M. Lyte, and R. P. A. Gaykema, "Infectioninduced viscerosensory signals from the gut enhance anxiety: implications for psychoneuroimmunology," Brain, Behavior, and Immunity, vol. 21, no. 6, pp. 721-726, 2007.

[82] D. F. MacFabe, D. P. Cain, K. Rodriguez-Capote et al., "Neurobiological effects of intraventricular propionic acid in rats: possible role of short chain fatty acids on the pathogenesis and characteristics of autism spectrum disorders," Behavioural Brain Research, vol. 176, no. 1, pp. 149-169, 2007.

[83] S. R. Shultz, D. F. MacFabe, K. P. Ossenkopp et al., "Intracerebroventricular injection of propionic acid, an enteric bacterial metabolic end-product, impairs social behavior in the rat: implications for an animal model of autism," Neuropharmacology, vol. 54, no. 6, pp. 901-911, 2008.

[84] E. Emanuele, P. Orsi, M. Boso et al., "Low-grade endotoxemia in patients with severe autism," Neuroscience Letters, vol. 471, no. 3, pp. 162-165, 2010.

[85] L. Desbonnet, L. Garrett, G. Clarke, J. Bienenstock, and T. G. Dinan, "The probiotic Bifidobacteria infantis: an assessment of potential antidepressant properties in the rat," Journal of Psychiatric Research, vol. 43, no. 2, pp. 164-174, 2008.

[86] D. Benton, C. Williams, and A. Brown, "Impact of consuming a milk drink containing a probiotic on mood and cognition," European Journal of Clinical Nutrition, vol. 61, no. 3, pp. 355361, 2007.

[87] A.V. Rao, A. C. Bested, T. M. Beaulne et al., "A randomized, double-blind, placebo-controlled pilot study of a probiotic in emotional symptoms of chronic fatigue syndrome," Gut Pathogens, vol. 1, no. 1, article 6, 2009.

[88] E. Slack, S. Hapfelmeier, B. Stecher et al., "Innate and adaptive immunity cooperate flexibly to maintain host-microbiota mutualism," Science, vol. 325, no. 5940, pp. 617-620, 2009. 


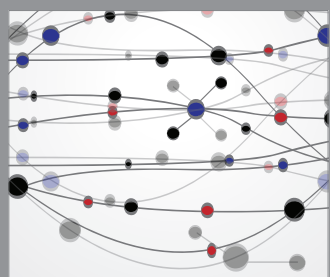

The Scientific World Journal
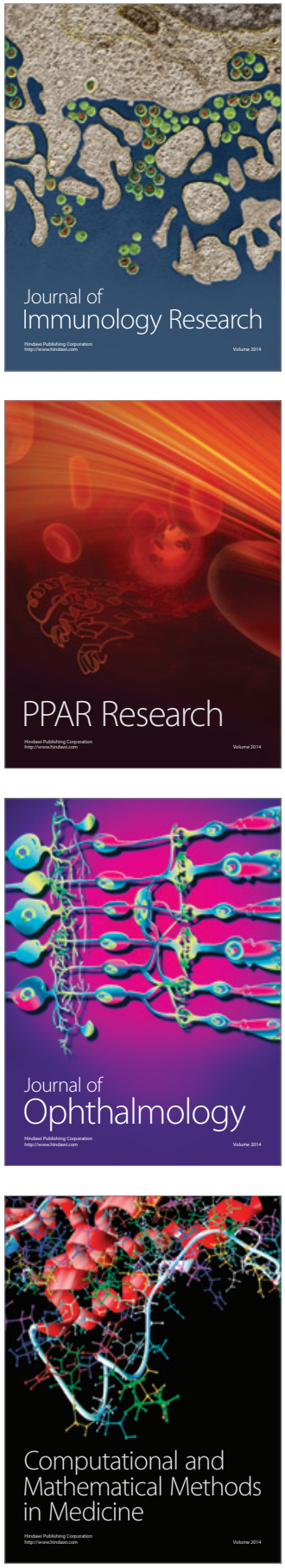

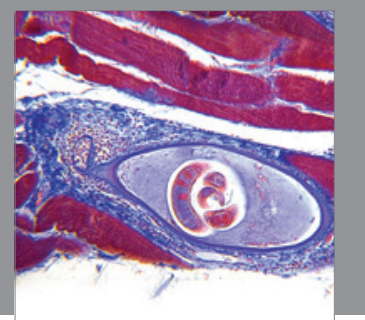

Gastroenterology

Research and Practice
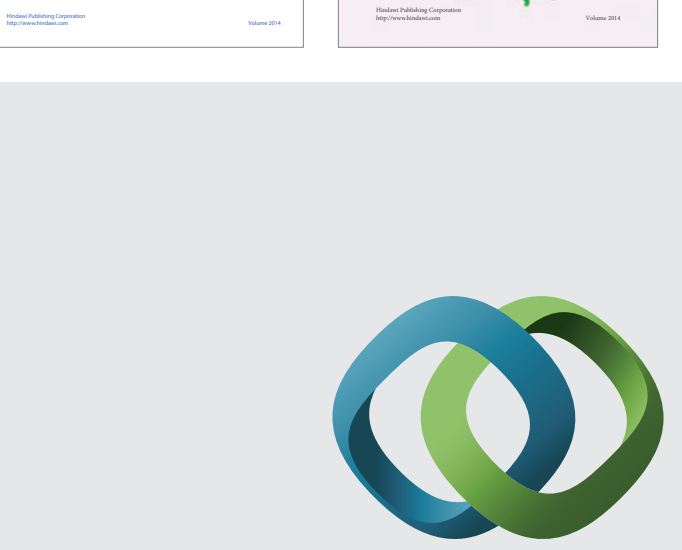

\section{Hindawi}

Submit your manuscripts at

http://www.hindawi.com
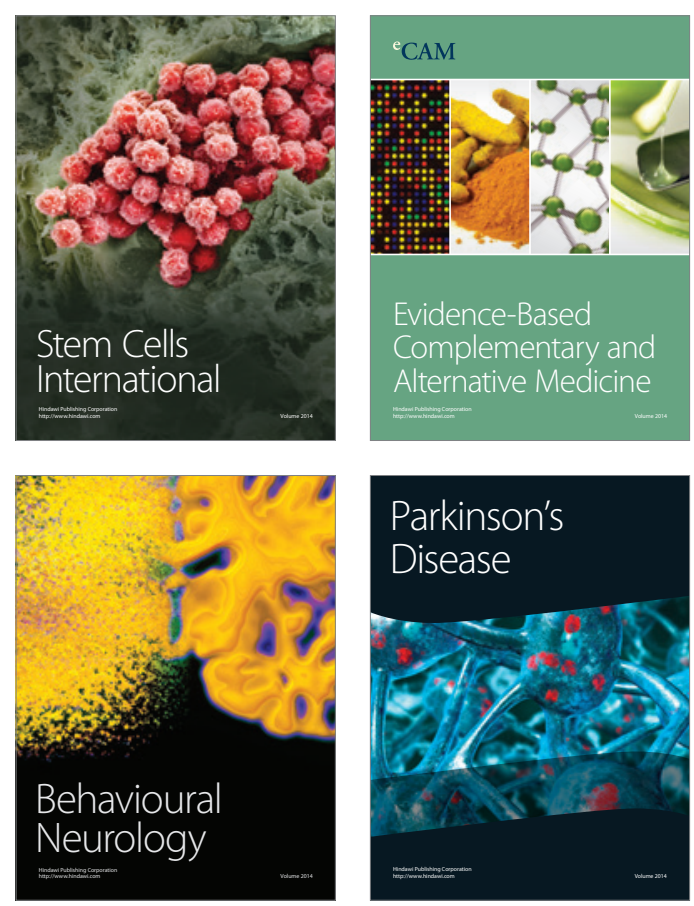

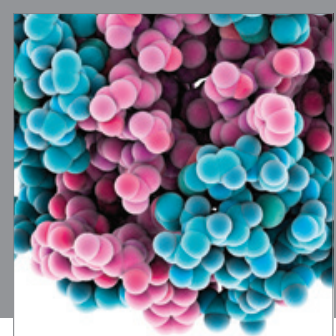

Journal of
Diabetes Research

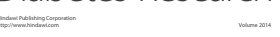

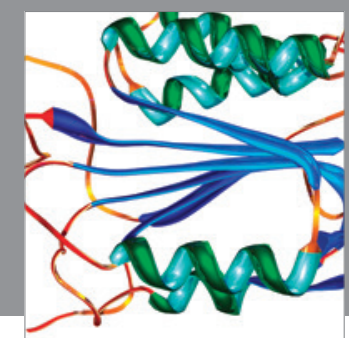

Disease Markers
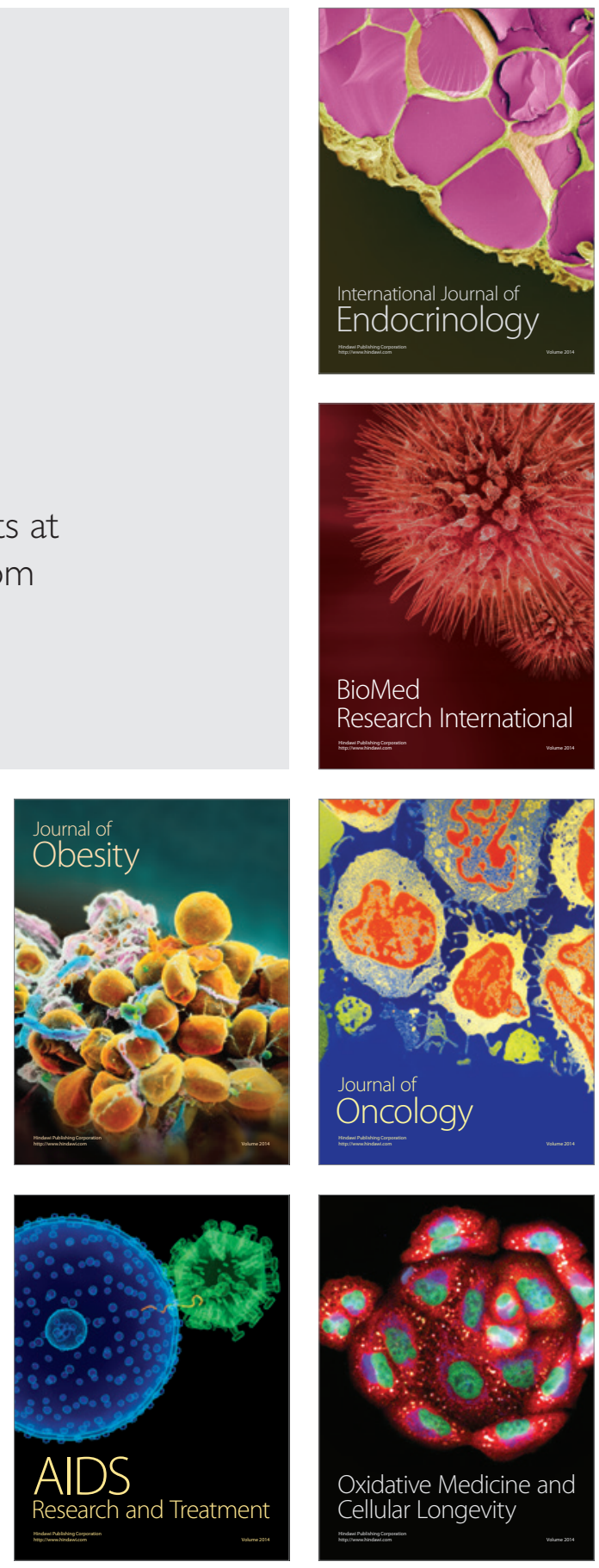